\title{
Interfacial resonant tunneling induced by folded bands and providing highly spin-polarized current in spinel-oxide barrier junctions
}

\author{
Kenji Nawa $\odot,{ }^{1, *}$ Keisuke Masuda $\odot,{ }^{1}$ and Yoshio Miura $\odot^{1,2}$ \\ ${ }^{1}$ Research Center for Magnetic and Spintronic Materials, National Institute for Materials Science (NIMS), \\ 1-2-1 Sengen, Tsukuba 305-0047, Japan \\ ${ }^{2}$ Center for Spintronics Research Network (CSRN), Graduate School of Engineering Science, Osaka University, \\ 1-3 Machikaneyama, Toyonaka, Osaka 560-8531, Japan
}

(Received 17 August 2020; revised 22 September 2020; accepted 30 September 2020; published 15 October 2020)

\begin{abstract}
In spintronics, simultaneous realization of high tunneling magnetoresistance and low resistivity in magnetic tunnel junctions (MTJs) is challenging because insulating layers with higher barrier heights generally generate highly spin-polarized currents but increase resistivity. We overcome this trade-off relationship using Brillouinzone-folded bands at the interfaces in the $\mathrm{Fe} /$ spinel $\mathrm{MgGa}_{2} \mathrm{O}_{4} / \mathrm{Fe}$ MTJ. Interfacial resonant states that enhance conductance are formed by folded bands, with $\mathrm{Fe}-\mathrm{O}$ hybridization playing a key role in the resonant effect intensity at the Fermi level. The electronegativity of cation Ga in spinel oxide $\mathrm{MgGa}_{2} \mathrm{O}_{4}$ is found to be a crucial physical quantity to control an intensity of the interfacial-resonant effect from the comparative analysis with the $\mathrm{MgAl}_{2} \mathrm{O}_{4}$-based MTJ.
\end{abstract}

DOI: 10.1103/PhysRevB.102.144423

\section{INTRODUCTION}

Spin transport, which originates from two-dimensional (2D) electronic structures, has the potential to enhance spinbased phenomena [1]. In the spintronics field, one important goal is the establishment of a method to realize a high magnetoresistance (MR) ratio and a low resistance-area product (RA) simultaneously for spin applications to read and write high-density stored data efficiently using low currents [2]. The tunneling-MR (TMR) effect occurs in artificial ferromagnetic multilayers that are separated by an insulator layer. High TMR is expected to occur in magnetic tunneling junctions (MTJs) containing well-crystallized insulators with a wide band gap. However, this results in a higher RA due to the increased barrier height [3,4]. Therefore, from a fundamental physics viewpoint, there is special interest in ways to break the tradeoff relationship described above. To date, several approaches have been used in attempts to overcome this situation [5-16], but further breakthroughs are required. Here, we propose a strategy to achieve high TMR and low RA simultaneously using folded-band engineering at the interface in an MTJ.

Spinel-type oxides, $A B_{2} \mathrm{O}_{4}$, offer rich controllability in their structural and electronic properties via selection of their constituent cations and compositions. Sukegawa, Miura, and their collaborators have discovered and explored the capabilities of spinel-oxide-barrier MTJs using experimental and

\footnotetext{
*nawa.kenji@nims.go.jp
}

Published by the American Physical Society under the terms of the Creative Commons Attribution 4.0 International license. Further distribution of this work must maintain attribution to the author(s) and the published article's title, journal citation, and DOI. theoretical techniques [17-24]. $\mathrm{MgAl}_{2} \mathrm{O}_{4}$ (MAO) is advantageous in offering a small lattice mismatch with bcc-based ferromagnetic materials. However, despite the realization of a full-epitaxial $\mathrm{Fe}(001) / \mathrm{MAO}$ interface without misfit dislocations, rather low TMR was observed experimentally [18]. This low TMR is caused by the bulklike band-folding (BF) effect, where folding of the bulk Fe band along the in-plane direction occurs because the lattice spacing of MAO is twice as large as that of Fe. This causes a new conducting channel to be opened in the minority-spin states and leads to the TMR reduction [17]. However, higher TMR of more than $400 \%$ was observed in subsequent experiments. This increase was understood to be caused by atomic disordering in the cation sites of MAO, which halves the effective lattice constant and suppresses both the BF effect and the appearance of the minority-spin states [18]. Very recently, this disordering effect was investigated further using combined $a b$ initio and machine-learning approaches [24].

Importantly, the BF effect occurs even at the interface. Figure 1 illustrates the 2D surface states of the majority spin at the $\mathrm{Fe}(001)$ surface. In the $2 \mathrm{D}$ primitive $\mathrm{Fe}$ cell $\left[a=a_{\mathrm{Fe}}\right.$; Fig. 1(a)], we see the density of states (DOS) characterized by the $\Gamma$-centered diamond shape and the DOSs near the Brillouin-zone (BZ) boundaries on $k_{x}=0$ and $k_{y}=0$, i.e., where $\left(k_{x}, k_{y}\right)=( \pm \pi / a, 0)$ and $(0, \pm \pi / a)$, but no such states appear around $\Gamma$ [Fig. 1(b)]. In contrast, an Fe supercell with a doubled in-plane lattice constant $\left(a=2 a_{\mathrm{Fe}}\right)$ has the $\mathrm{BZ}$ shrunk to one quarter of the original size. The electronic structures outside the first $\mathrm{BZ}$ are folded with respect to the zone edges and additional states are thus formed around $\Gamma$ [Fig. 1(c)], although no minority-spin states are found around $\Gamma$. These results indicate that the zone-folded 2D bands appear at the $\mathrm{Fe}(001) /$ spinel interface and that the folded bands of $\mathrm{Fe}$ can be connected with the evanescent states of the unfolded 
(a)

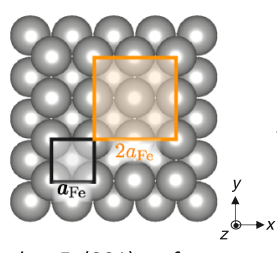

bcc Fe(001) surface (b)

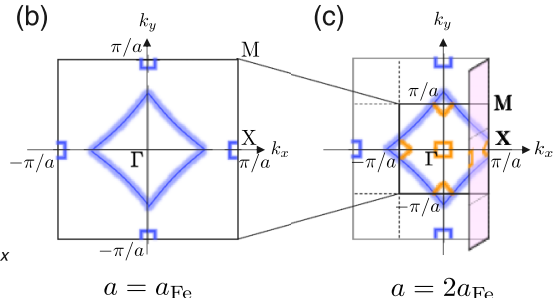

FIG. 1. (a) Top view of $\mathrm{Fe}(001)$ surface showing the primitive $\left(a=a_{\mathrm{Fe}}\right)$ and doubled $\left(2 a_{\mathrm{Fe}}\right)$ in-plane lattice constants. Illustrations of the majority-spin surface DOS of the (b) primitive and (c) doubled lattices in the 2D BZ. The first BZ in (c) is indicated by the bold line. (See Appendix A for the detailed calculation results.)

spinel bands, which then contributes to the spin-dependent tunneling conductance.

In this paper, we demonstrate that both high TMR and low RA can be obtained simultaneously through interfacial resonant tunneling in the majority spin caused by the BF effect at the $\mathrm{Fe} / \mathrm{MgGa}_{2} \mathrm{O}_{4}(\mathrm{MGO})(001)$ interface. This interfacial resonant (IR) effect is induced by the folded-band structure at the interface, which enhances the transmittance significantly. A similar phenomenon occurs in the Fe/MAO/Fe MTJs, but the contribution of the IR effect to the TMR is not as large in this case. Interestingly, we find that the BF effect induces a competition between the positive and negative contributions to the TMR. The former is the IR tunneling in the majority spin, while the latter, as reported previously [17], is the bulklike coherent tunneling in the minority spin. We clarify that the intensity of the IR effect originates from the electronegativities of the cations of $\mathrm{Ga}$ and $\mathrm{Al}$. Our results, thus, open a pathway toward the successful development of MTJ devices with high TMR and low RA and toward the engineering of 2D magnetism via the BF effect by appropriately selecting the $B$ element in $\mathrm{Mg}$-based spinel oxides $\mathrm{MgB}{ }_{2} \mathrm{O}_{4}$.

We mention that the experimental fabrication of the MGObased MTJ has been reported so far. Because the MGO bulk crystal has an energy band gap of $4.9 \mathrm{eV}$ [25] that is smaller than those of MAO $(7.8 \mathrm{eV})[26,27]$ and rock-salt $\mathrm{MgO}$ $(7.58-7.8 \mathrm{eV})[28,29]$, a lower barrier height is thus expected in the MTJ with the MGO tunneling barrier. Experimentally, an RA of $\sim 10^{2} \Omega \mu \mathrm{m}^{2}$ (for a barrier thickness of $\sim 1.8 \mathrm{~nm}$ ) has been obtained in an $\mathrm{Fe} / \mathrm{MGO} / \mathrm{Fe}$ MTJ [30]. This value is several orders of magnitude smaller than the reported values for Fe/MAO/Fe MTJs [20,30], without simultaneously sacrificing the TMR value; a TMR of $196 \%(121 \%)$ at low temperature (room temperature) was reported in a MGO-MTJ while a value of $165 \%(117 \%)$ was reported in a MAO-MTJ. However, there have been no theoretical reports on the spindependent transport properties of MGO-based MTJs to date.

\section{MODEL AND METHOD}

To model the MGO-MTJ, we prepared $n$ monolayers (MLs) $(n=9 \sim 25)$ of normal-type spinel MGO sandwiched between five MLs of $\mathrm{Fe}$ with the in-plane lattice constant fixed at $5.733 \AA$, which corresponds to a 45-degree rotation of the MGO unit cell and the doubled Fe lattice [Fig. 2(a)]. The in-plane lattice constant we used is consistent with that in previously reported experiments [18,20,22,30]. For the MGObased MTJ, we confirmed that the Ga-site termination with $\mathrm{O}$ on top of the Fe configuration is energetically favorable, as consistent with the MAO-MTJ reported previously [17].

The self-consistent electronic structures were computed using the ultrasoft pseudopotential plane-wave method implemented in the QUANTUM ESPRESSO code [31-33] using the generalized gradient approximation for the exchange correlation [34]. Cutoff energies of 30 and 300 Ry were used for the wave functions and the charge density, respectively. The BZ integration was performed using a $10 \times 10 \times 1$

(a)
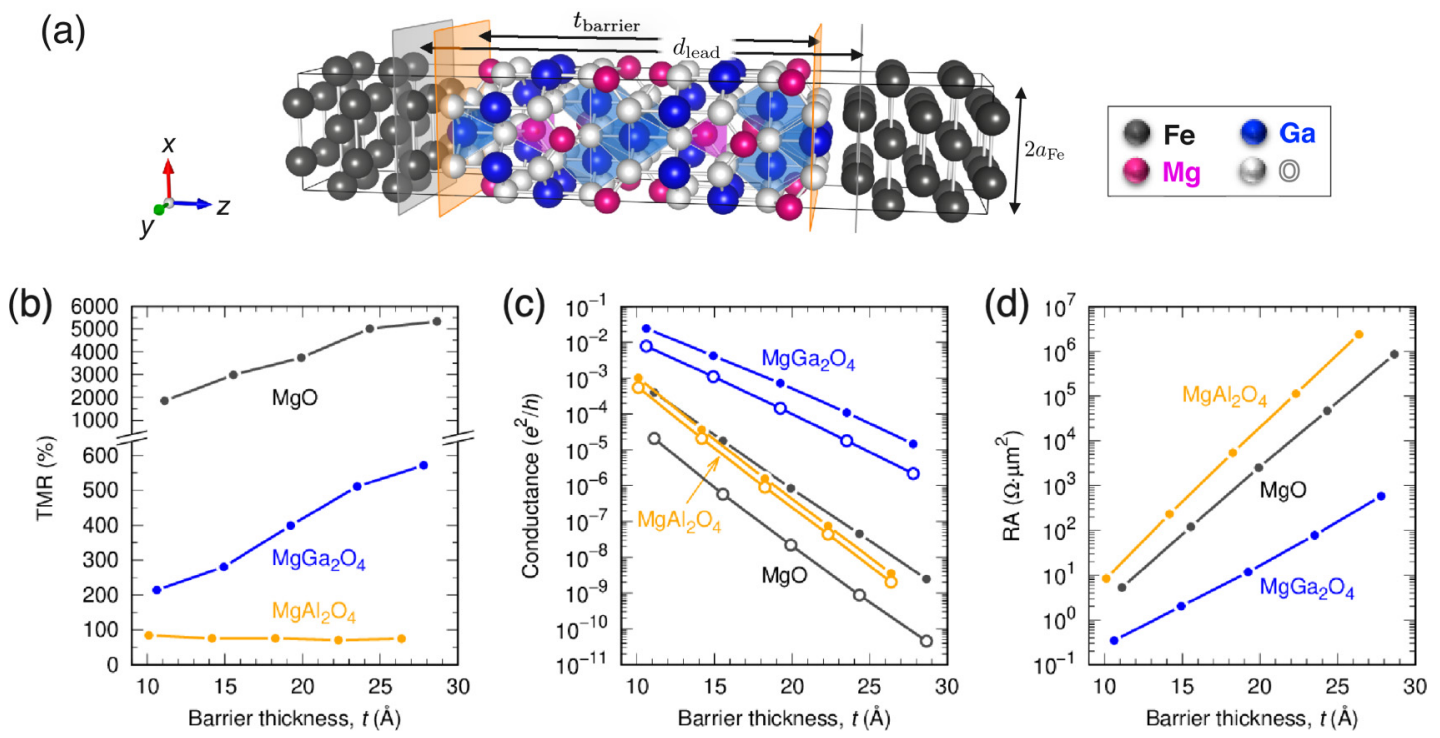

FIG. 2. (a) MTJ structure composed of $\mathrm{Fe}(5) / \mathrm{MGO}(n) / \mathrm{Fe}(5)$, where the number of layers of each material is given in parentheses $(n=$ 9 25). Barrier thickness dependencies of (b) TMR, (c) tunneling conductance, and (d) RA for MTJs containing MGO (blue). Results for MTJs containing MAO (orange) and rock-salt MgO (black) are also shown for reference. In (c), closed (open) plots represent parallel (antiparallel) magnetization condition. 
Monkhorst-Pack special $k$-point mesh. Full relaxation of all the atomic positions was carried out until the forces acting on each atom were minimized to less than $10^{-3} \mathrm{eV} / \AA$.

The conductances were obtained by solving the scattering equation with infinite boundary conditions where the scattering wave functions and their derivatives are connected to the Bloch states of electrodes. The details are described in Refs. [31,35]. The optimistic TMR ratio $\left(G_{\mathrm{P}}-G_{\mathrm{AP}}\right) / G_{\mathrm{AP}} \times$ $100(\%)$ was used, where $G_{\mathrm{P}}$ and $G_{\mathrm{AP}}$ stand for the conductances of the parallel and antiparallel magnetizations, respectively, and the RA was evaluated from the parallel state.

\section{RESULTS AND DISCUSSION}

Figures 2(b)-2(d) summarize the barrier thickness $(t)$ dependencies of the spin-dependent transport properties through comparisons with the MTJs containing the MAO and rocksalt $\mathrm{MgO}$ barriers. (See Ref. [36] for the barrier thickness definition.) The TMR ratio for the MGO-MTJ is $214 \%$ at $t=10.6 \AA(n=9)$ and increases to $572 \%$ as the barrier thickness increases ( $t \sim 26.3 \AA$; $n=25)$, as shown in Fig. 2(b). Figure 2(c) shows that the conductance of the MGO-MTJ with parallel magnetization decays more slowly than that with antiparallel magnetization, leading to the increase in the TMR. This is similar to the characteristics of the rock-salt MgO-MTJ [37]. In contrast, the TMR in the MAO-MTJ remains almost constant at $\sim 75 \%$; this occurs because the decays in the MTJ conductances are almost the same for both the parallel and antiparallel magnetizations. Because the MGO-MTJ shows the highest conductance among the models investigated here, the lowest RA is obtained at each of the barrier thicknesses [Fig. 2(d)].

A major contribution to the spin-dependent transmittance in the MGO-MTJ can be found in the remarkably high peaks of the majority-spin states in the parallel magnetization case at the transverse wave vector $\mathbf{k}_{\|}=(0, \pm 0.06)$, i.e., slightly off the $\Gamma$ [Fig. 3(a)]. This indicates that the "coherent" tunneling that occurs at $\Gamma$ may not be the central origin of the TMR, as was expected from the complex band calculation results for bulk MGO (see also Appendix B). Figure 3(c) shows the calculated projected tunneling DOS (PTDOS) for the local atomic orbitals of $\mathrm{O}$ and $\mathrm{Ga}$ at the Fermi level $\left(\left|c_{\alpha \ell m}\right|^{2}\right)$ at $\mathbf{k}_{\|}=(0, \pm 0.06)$. Here, $c_{\alpha \ell m}$ are the coefficients of the scattering wave functions of the orbital and magnetic quantum numbers $\ell$ and $m$, respectively, at the atom $\alpha$ [35]. We find that the dominant contributions come from the $s, p_{z}$, and $d_{z^{2}}$ orbitals. These states are characterized as the $\tilde{\Delta}_{1}$ symmetry at $\mathbf{k}_{\|}=(0,0)$ and are responsible for the giant TMR that occurs in bcc-based electrode MTJs [17,18,38-42], so we refer to the $\tilde{\Delta}_{1}^{*}$ symmetry hereafter. The magnitudes for the other orbitals are all approximately zero (the results are not shown). Additionally, a notable feature that can be observed in Fig. 3(c) is that the PTDOS values are enhanced and show a $v$-shaped structure on the right-hand side of the barrier, while in contrast, they decay monotonically on the left-hand side. This reflects the existence of the IR state [38]. A very large wave-function amplitude appears at the interfaces, even for electrons that are outgoing from the barrier region, and this results in a large conductance for the majority-spin state. The
MGO

(a)

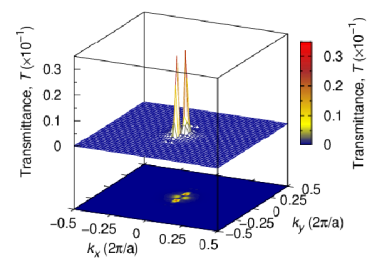

(c)

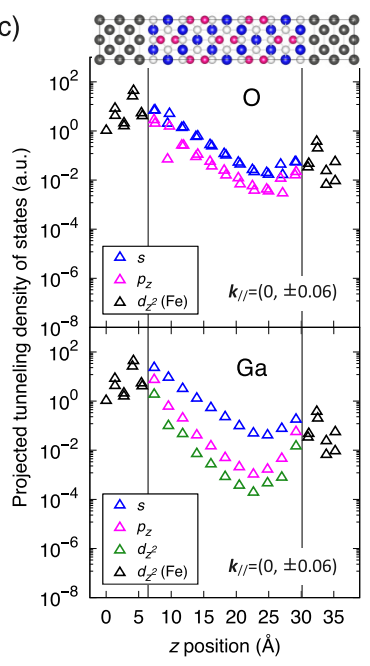

MAO

(b)

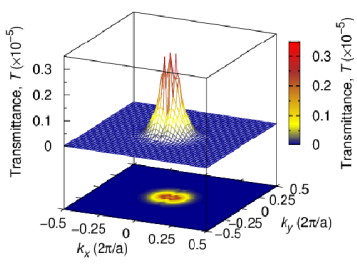

(d)
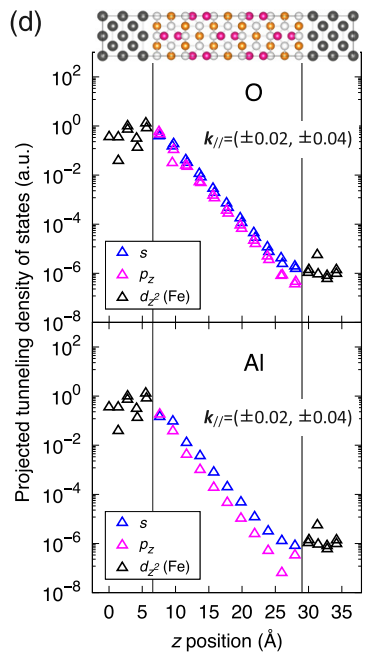

FIG. 3. (a) Majority spin transmittance in parallel magnetization in $\mathbf{k}_{\|}$space and (c) PTDOS for the $\tilde{\Delta}_{1}^{*}$ states as a function of the $z$ position of the supercell at $\mathbf{k}_{\|}=(0, \pm 0.06)$ in the MGO-MTJ $(n=25)$. (b), (d) The same for the MAO-MTJ but with (d) at $\mathbf{k}_{\|}=$ $( \pm 0.02, \pm 0.04)$.

absolute value of the PTDOS is the highest in the $s$ orbital, but the values in the $p_{z}$ and $d_{z^{2}}$ orbitals are not negligible. We also stress that the increase in the PTDOS begins at the $\mathrm{Ga}_{2} \mathrm{O}_{4}$ layer of the MGO located third or fourth from the right interface, regardless of the barrier thickness. The low RA mainly originates from the majority-spin resonant states rather than from the intrinsic small band gap of MGO.

In the MAO-MTJ, as shown in Fig. 3(b), the highest but rather broad transmittance peaks are obtained at $\mathbf{k}_{\|}=$ $( \pm 0.02, \pm 0.04)$, and relatively large transmittance is found at $\Gamma$ because of $\tilde{\Delta}_{1}$-symmetric coherent tunneling [17,43]. Figure 3(d) shows the rapid decays of the $\tilde{\Delta}_{1}^{*}$ PTDOS. An increase in the PTDOS can be seen at the right interface, but the magnitude of this increase is quite small because the IR effect is weaker than that in the MGO-MTJ, as we discuss later. Apparently, the decay of the conductance in the parallel magnetization case is not suppressed when compared with that in the antiparallel case, as indicated in Fig. 2(c). Note that for the antiparallel magnetization case, a broad transmittance peak is found at $\Gamma$ in both the MGO- and MAO-MTJs; this is consistent with previously reported results [17].

To clarify the critical difference in the significance of the IR effect between the MGO- and MAO-MTJs, layerby-layer electronic DOS summed over the $\tilde{\Delta}_{1}^{*}$ bands are presented in Fig. 4. In the MGO-MTJ, a twofold symmetric DOS that occurs around $\Gamma$ is visible in both bulk and interface Fe [Figs. 4(a) and 4(b)], where the original fourfold 

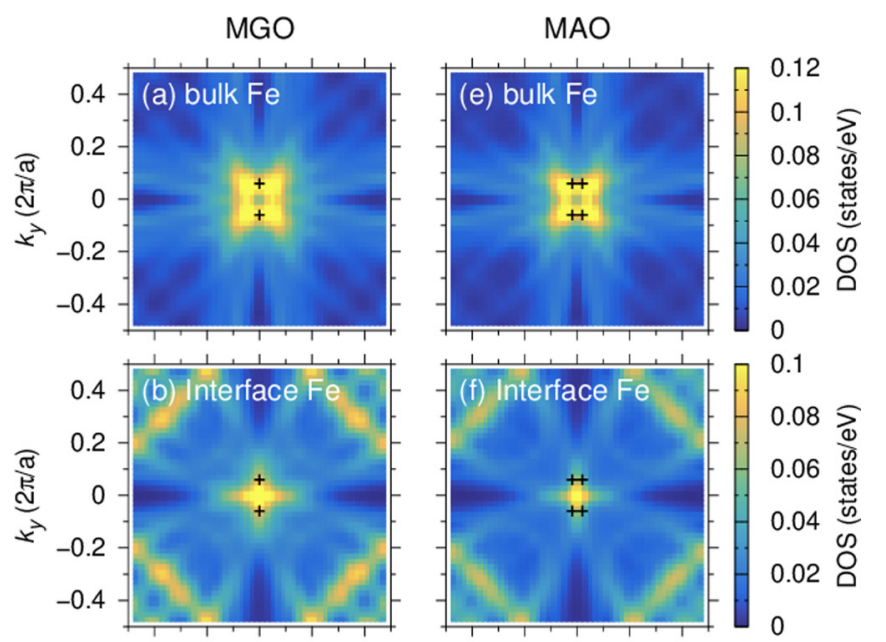

0.1

$0.08>$

0.06 के

$0.04 \frac{\pi}{\infty}$

0.02 ○
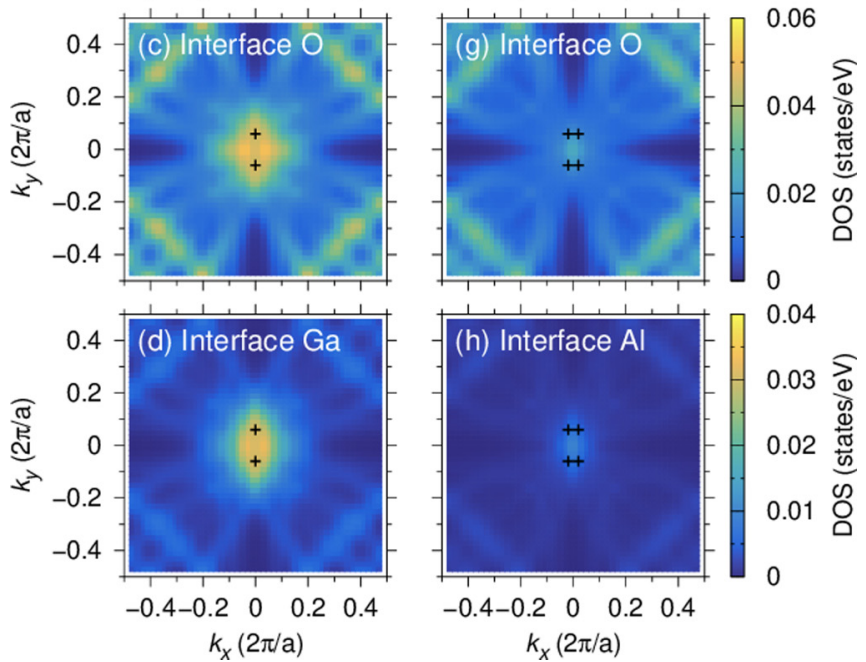

FIG. 4. Layer-by-layer electronic DOS summed over the $\tilde{\Delta}_{1}^{*}$ states ( $s, p_{z}$, and $d_{z^{2}}$ states) in the 2D BZ for the MGO-MTJ; (a) bulk $\mathrm{Fe}$, and (b) interface $\mathrm{Fe}$, (c) $\mathrm{O}$, and (d) Ga. (e)-(h) The same for the MAO-MTJ with the exception of (h) interface Al. Cross symbols indicate the $\mathbf{k}_{\|}$vectors that show the largest transmittances.

symmetry is reduced by attaching the spinel structure. The $\mathbf{k}_{\|}=(0, \pm 0.06)$ points showing the huge resonant transmittances are found in the DOS, in which the interfacial states of both $\mathrm{O}$ and $\mathrm{Ga}$ exist [see the cross symbols in Figs. 4(c) and 4(d)]. Importantly, these DOS only appear at the interface and are constructed via the BF effect, as illustrated in Fig. 1. In contrast, in MAO, although a similar DOS distribution can be seen in the bulk Fe [Fig. 4(e)], tiny surface states remain at $\mathbf{k}_{\|}=( \pm 0.02, \pm 0.06)$ [Figs. 4(f)-4(h)]. Therefore, the intensities of the IR peaks in the tunneling conductance are proportional to the magnitude of the interfacial DOS. The interfacial electronic bands are found near the corners of the 2D BZ of the interface Fe and O [Figs. 4(b), 4(c) 4(f), and 4(g)], but zero transmittance is observed at these $\mathbf{k}_{\|}$[Figs. 3(a) and $3(\mathrm{~b})]$. This is caused by an absence of the $\tilde{\Delta}_{1}^{*}$ states in the bulklike Fe in these regions; the surface states, which are not connected to the tunneling channel in the bulk Fe, no longer propagate inside the barrier [44].

In Fig. 5, we show transmittances as a function of the energy relative to the Fermi level for the MGO-MTJ at $\left(k_{x}, k_{y}\right)=$
$(0, \pm 0.06)$ and the MAO-MTJ at $\left(k_{x}, k_{y}\right)=( \pm 0.02, \pm 0.04)$ with the corresponding band dispersions along $k_{z}$ direction. In the MGO-MTJ, a strong sensitivity of the IR transmittance is evident from Figs. 5(a) and 5(b). The transmittance is majorly attributed to the $\tilde{\Delta}_{1}^{*}$ band [43]. Interestingly, a high transmittance is peaked at the Fermi energy [Fig. 5(a)] as a result of the contributions from the $\tilde{\Delta}_{1}^{*}$ state [Fig. 5(b)]. Even at the same valence $\tilde{\Delta}_{1}^{*}$ band, a reduction of the transmittance is found at $0.08 \mathrm{eV}$ below the Fermi energy due to a small magnitude of the transmittance at this energy level. These indicate that a shift of the Fermi energy induced by interfacial defects may affect the IR state adversely, which is similar to the $\mathrm{Fe} / \mathrm{MgO} / \mathrm{Fe} \mathrm{MTJ}$ system [45-47]. This transmittance behavior is a characteristic feature found at the $\left(k_{x}, k_{y}\right)=$ $(0, \pm 0.06)$ point, while a similar observation is not confirmed at $\Gamma$. Note that another transmittance drop can be seen at $\sim 0.05 \mathrm{eV}$ above the Fermi level which corresponds to the zone-edged $\tilde{\Delta}_{1}^{*}$ band, as shown in Fig. 5(b). In contrast, in the MAO-MTJ with Figs. 5(c) and 5(d), a sharp profile of the transmittance originating from the resonant states is visible around the Fermi energy but is insignificant as expected from the discussions in Figs. 3(b) and 3(d).

Because $\mathrm{O}$ and $\mathrm{Fe}$ form the on-top configuration at the interface, we see strong hybridization between the Fe $d_{z^{2}}$ and $\mathrm{O} p_{z}$ orbitals [Fig. 6(a)]. The bonding and antibonding states in the majority spin are located 6 and $1 \mathrm{eV}$ below the Fermi energy, respectively. Furthermore, the insets of Fig. 6(a) show that the local DOS in the MGO-MTJ is much larger than that in the MAO-MTJ at the Fermi level. This difference arises from the strength of the $\mathrm{Fe} d_{z^{2}}-\mathrm{O} p_{z}$ hybridization, which we finally discuss here. In a spinel-based MTJ, the $\mathrm{O}$ is slightly shifted from being exactly on top of the $\mathrm{Fe}$ because of the cation distributions. The polarity given by the electronegativity difference between $\mathrm{Ga}(\chi=1.81)$ and $\mathrm{O}$ (3.44) of $\delta \chi=1.63$ is smaller than that between $\mathrm{Al}$ (1.61) and $\mathrm{O}$, where $\delta \chi=1.83$ [48]. These different polarities cause the ionic bond length of $\mathrm{Ga}-\mathrm{O}$ to be larger than the corresponding $\mathrm{Al}-\mathrm{O}$ length, thus meaning that the shift of $\mathrm{O}$ from the $\mathrm{Fe}$ on-top position at the Fe/MGO interface is smaller $(0.228 \AA)$ than that at the $\mathrm{Fe} / \mathrm{MAO}$ interface (0.323 $\AA$ ) [Fig. 6(b)]. Consequently, as illustrated in Fig. 6(c), the energy gap between the bonding $p_{z}$ and antibonding $d_{z^{2}}$ states at the Fe/MGO interface becomes wider than that at the Fe/MAO interface [49]. These results indicate that the cation $B$ element possessing a large electronegativity tends to strengthen the intensity of the IR state at the $\mathrm{Fe} /$ spinel interface.

We here mention that the IR effect has been paid considerable attention previously in mostly $\mathrm{MgO}$-based MTJs [38,44,50-55]. However, its contribution to the TMR is not as significant because the resonant states only appear in the minority spin in the $\mathrm{Fe} / \mathrm{MgO} / \mathrm{Fe}$ MTJ. Furthermore, the appearance of IR-effect-induced tunneling at the Fermi level is quite sensitive to the ferromagnetic/insulating layer thicknesses [44,56] and the in-plane lattice spacing [57,58], although these conductances can be tuned via application of finite bias voltages $[59,60]$ and insertion of extra transitionmetal layers at the interface [56]. Therefore, we emphasize here that the IR effect on the TMR in the MGO-based MTJ is superior to that in the MgO-based MTJ. 

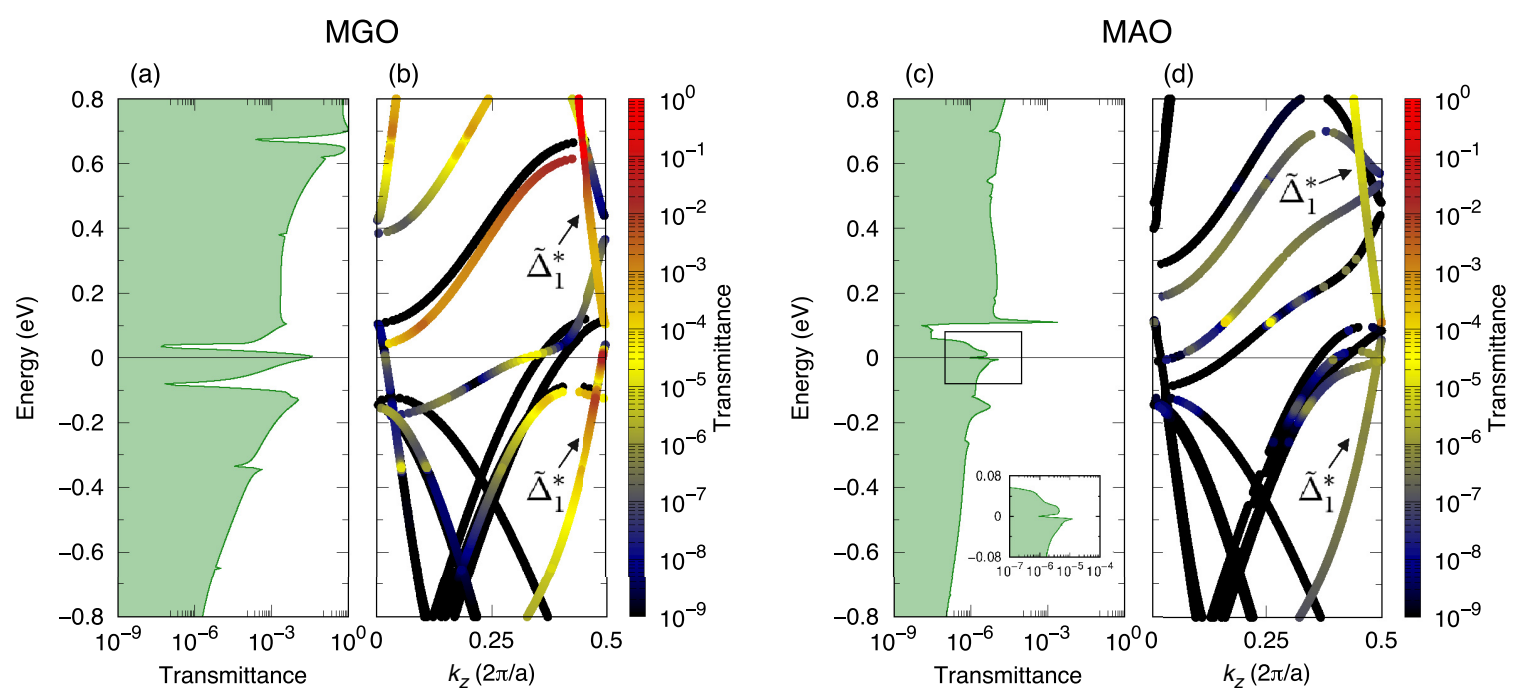

FIG. 5. (a) Transmittance against the energy in majority-spin state at $\left(k_{x}, k_{y}\right)=(0, \pm 0.06)$ and (b) corresponding band structure along $k_{z}$ direction, where transmittance is projected, for the MGO-MTJ with parallel magnetization configuration. (c), (d) The same for the MAO-MTJ but at $\left(k_{x}, k_{y}\right)=( \pm 0.02, \pm 0.04)$. The Fermi energy is set to zero. Arrows in (b) and (d) specify the $\tilde{\Delta}_{1}^{*}$ band giving large transmittance. Inset in (c) focuses on the Fermi energy.

\section{SUMMARY}

In summary, we have clarified theoretically that the IR tunneling caused by the BF effect in the majority-spin state is an important factor in breaking the trade-off relationship between the high TMR and the low RA in MTJs containing the spinel-type oxide tunneling barrier. Atomic-orbital hybridization between the $\mathrm{Fe}-d$ and $\mathrm{O}-p$ states plays an important role in determining the intensity of the resonant state. Thus, the intensity of the IR state in the MTJ with a $\mathrm{MgGa}_{2} \mathrm{O}_{4}$ barrier is found to be stronger than that in the $\mathrm{MgAl}_{2} \mathrm{O}_{4}$ case, where the difference of electronegativities between constituent $\mathrm{O}$ and cation $B$ element ( $B=\mathrm{Ga}$ or $\mathrm{Al}$ ) in $\mathrm{Mg} B_{2} \mathrm{O}_{4}$ barrier is one of the essential factors to characterize the effect of interfacial resonance. A $b$ initio-predicted results open a way to control the IR state by choosing an appropriate cation element in the spinel oxide and invites experimental confirmation for realizing high-performance spintronics devices.

\section{ACKNOWLEDGMENTS}

The authors are grateful to H. Sukegawa and S. Mitani at NIMS and to T. Suzuki, S. Ichikawa, and K. Nakada
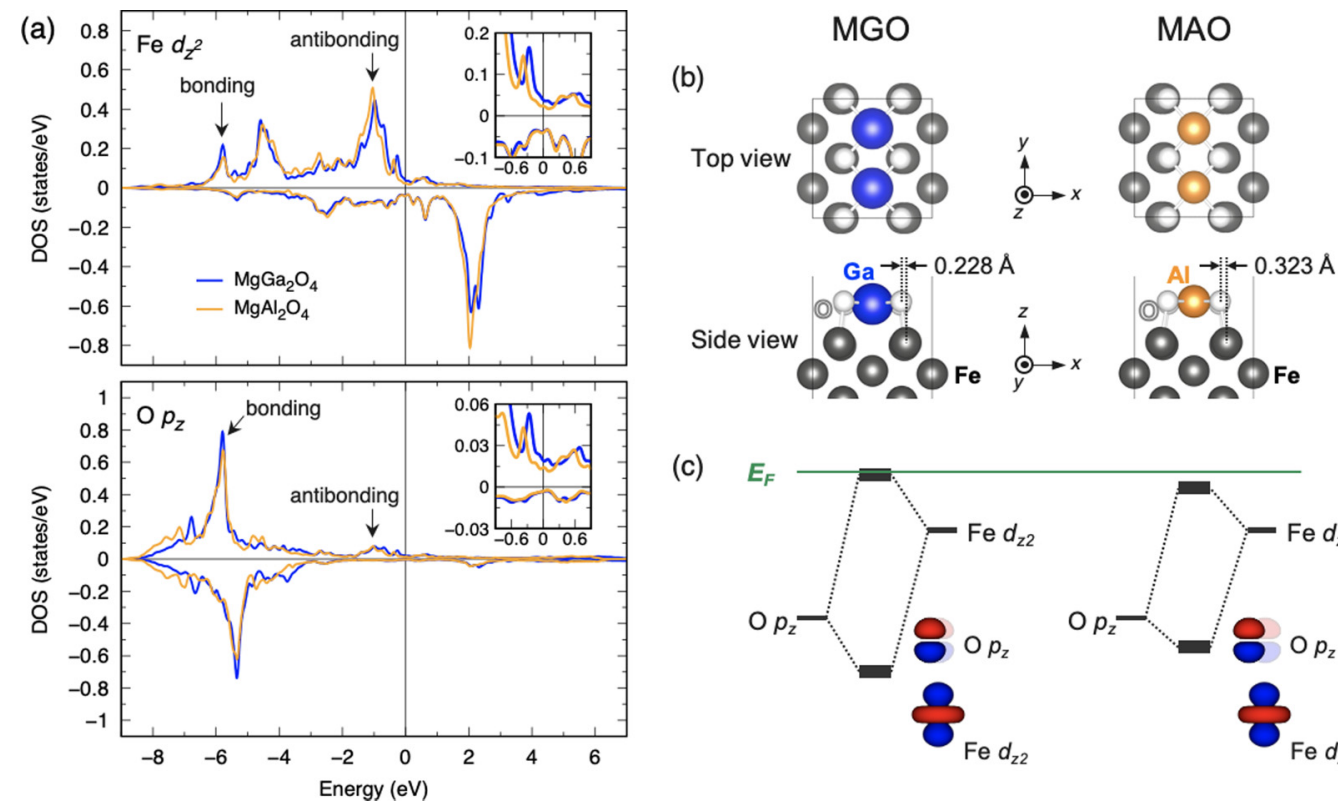

(c)

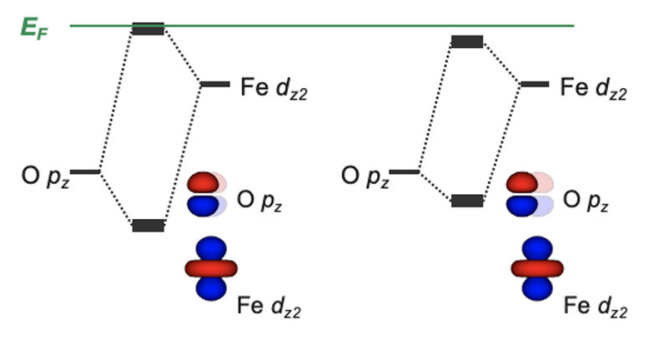

FIG. 6. (a) Electronic DOS for MTJs with MGO (blue) and MAO (orange) barriers projected on the interface Fe $d_{z^{2}}$ (top panel) and O $p_{z}$ (bottom panel), with insets focusing on the Fermi level. The Fermi energy is set to zero. (b) Top and side views of the interface structures. (c) Schematic energy diagram showing the hybridization between the on-top Fe $d_{z^{2}}$ and $\mathrm{O} p$. 
Surface state

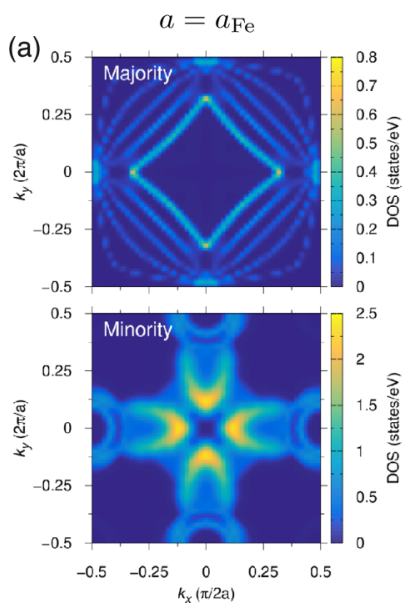



Bulk state

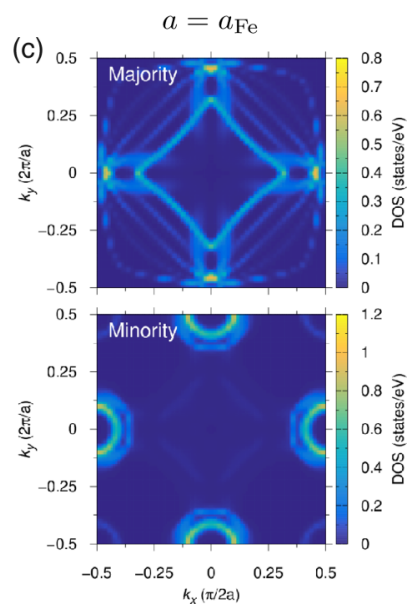

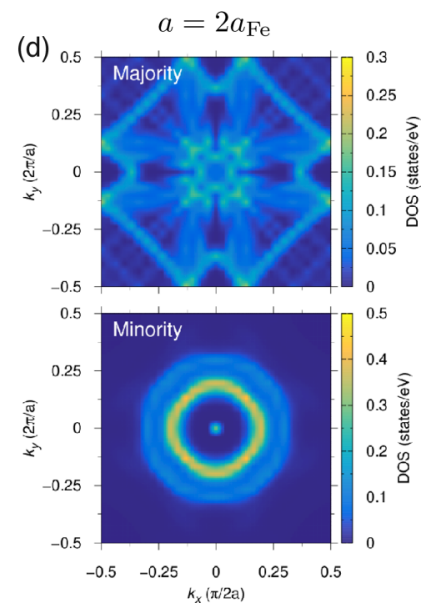

FIG. 7. Surface DOS plots in the first BZ for (a) primitive $\left(a=a_{\mathrm{Fe}}\right)$ and (b) doubled $\left(2 a_{\mathrm{Fe}}\right)$ in-plane lattice constants. Bulk DOS plots for the lattice constants of (c) $a_{\mathrm{Fe}}$ and (d) $2 a_{\mathrm{Fe}}$. The top and bottom panels are for the majority- and minority-spin states, respectively.

at TDK Corporation for fruitful discussions and suggestions. This work was supported in part by TDK Corporation and by Grants-in-Aid for Scientific Research (Grants No. JP16H06332, No. JP17H06152, and No. JP20H02190) from the Japan Society for the Promotion of Science. Computations were performed using the facilities of the Numerical Materials Simulator at NIMS and the Supercomputer Center at the Institute for Solid State Physics, the University of Tokyo.

\section{APPENDIX A: Fe(001) SURFACE CALCULATIONS}

For the calculation of the $\mathrm{Fe}(001)$ surface, two supercells consisting of nine fully relaxed MLs of bcc Fe and a vacuum region of $10 \AA$ were prepared, where the first had the primitive in-plane lattice constant $a=a_{\mathrm{Fe}}\left(a_{\mathrm{Fe}}=2.8665 \AA\right)$ and the other had the doubled lattice constant $a=2 a_{\mathrm{Fe}}$. A special $k$-point mesh with a $50 \times 50 \times 1(30 \times 30 \times 1)$ grid was used to calculate the 2D surface DOS of the primitive (supercell) Fe lattice.

Figure 7 presents the calculated 2D DOS. Note that only the $s, p_{z}$, and $d_{z^{2}}$ orbitals characterized by the $\Delta_{1}$ symmetry at the zone center $\Gamma$ were plotted because most of the tunneling electrons were contributed by these orbitals, as we discussed in the main text and as reported previously in the $\mathrm{Fe} / \mathrm{MAO} / \mathrm{Fe}$ MTJ $[17,18]$. Figures 1(b) and 1(c) in the main text illustrate the visible features found in the top parts of Figs. 7(a) and 7 (b), where the finite DOS was found around $\Gamma$ in the BZ of the doubled lattice because of the folded-band structures at the zone boundaries (full details are given in the main text). Importantly, no minority-spin states were folded around the $\Gamma$ point [bottom parts of Figs. 7(a) and 7(b)].

We also note that our calculations clearly reproduced the electronic structure of double-lattice $\mathrm{Fe}$ for bulk states that was reported previously [17]. For the minority-spin states, as shown in the bottom part of Fig. 7(d), a finite DOS can be seen at the $\Gamma$ point that was not found in the primitive $\mathrm{Fe}$ cell calculations [see Fig. 7(c), bottom part]. We confirm that these states cause a coherent tunneling channel in the minority states of the $\mathrm{Fe} /$ spinel/Fe MTJ.

\section{APPENDIX B: BULK BAND STRUCTURE FOR SPINEL MGO}

The spinel oxide, i.e., the so-called normal type, consists of the ordered cations $A$ and $B$ occupying the tetrahedral and octahedral sites, respectively, in an fcc $\mathrm{O}$ sublattice, corresponding to the space group $F d \overline{3} m$ (No. 227) [Fig. 8(a)]. An inverse-type structure also exists in which the tetrahedral site is occupied randomly by the cations $A$ and $B$. For MGO, a mixed-spinel ground state was reported in the experiments, where the degree of the inverse structure varied over a wide range, depending on the thermal equilibrium conditions in the experiments [61-65], whereas the inverse spinel structure was stable in the calculations [66].

In this paper, we assumed that MGO has a normal-type spinel structure and the results were then compared with those for the normal-spinel MAO MTJ. Previous theories reported that in the $\mathrm{Fe} /$ spinel/ $\mathrm{Fe}(001) \mathrm{MTJ}$, the on-top configuration of $\mathrm{O}$ and $\mathrm{Fe}$ at the interface played an essential role in the propagation of the tunneling wave functions $[17,18]$, as in the case of the well-known $\mathrm{Fe} / \mathrm{MgO} / \mathrm{Fe}$ MTJ $[38,39]$. However, the $\mathrm{O}$ anions in the spinel oxides were displaced from their ideal positions, where the bond lengths of the tetrahedral and octahedral sites are equivalent, depending on the cation distributions [67]. Therefore, the $\mathrm{O}$ atoms were slightly off the top of the $\mathrm{Fe}$ in the $x y$ plane at the $\mathrm{Fe} /$ spinel interface in the MTJ. The displacements of the anions at the interface cause a remarkable difference between the MGO- and MAO-MTJs (as discussed in the main text), and thus the assumption of the normal-spinel structure is significant with regard to the discussion of the structural and electronic complexities of the interface. It should be mentioned that in the $\mathrm{Fe} / \mathrm{MAO} / \mathrm{Fe}$ MTJ, the displaced anions modify the $\mathrm{Fe}-\mathrm{O}$ orbital hybridization from the on-top $\mathrm{Fe}-\mathrm{O}$ configuration (likely to be an $\mathrm{Fe} / \mathrm{MgO} / \mathrm{Fe} \mathrm{MTJ}$ ), and the underlying mechanism of the perpendicular magnetocrystalline anisotropy thus differs from that of the $\mathrm{Fe} / \mathrm{MgO}$ interface [68].

The real and imaginary band structures along the [001] direction were calculated from the tetragonal bulk lattice shown in Fig. 8(a) [the corresponding BZ is in Fig. 8(b)]. The 
(a)

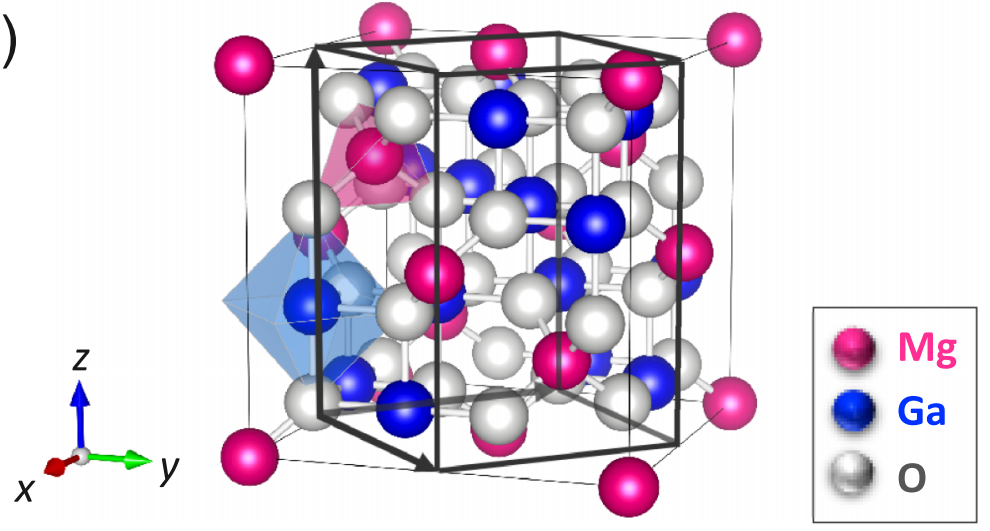

(b)

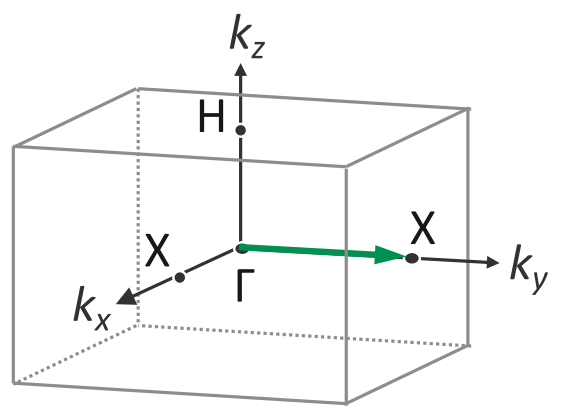

(c)

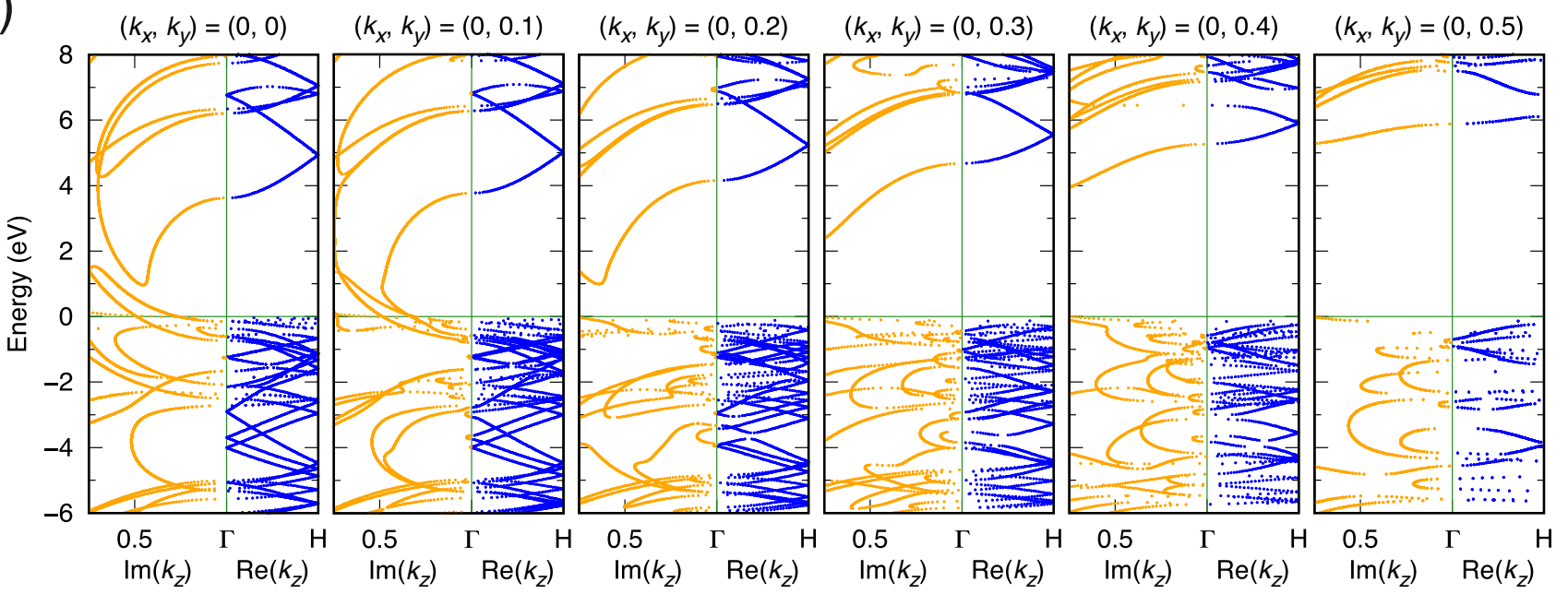

FIG. 8. (a) Geometry of the normal-spinel MGO: magenta, blue, and white balls represent the Mg, Ga, and O atoms, respectively. The tetragonal cell, for which the in-plane lattice constant is matched with the double constant of bcc Fe in the MTJ, is given by the black solid line with (b) the corresponding BZ. (c) Real (blue) and imaginary (yellow) band structures of tetragonal MGO along the [001] direction at the $\Gamma-X k$-path in the BZ.

spinel structure has $C_{2 v}$ symmetry along the conducting [001] direction, although it has $C_{4 v}$ symmetry in the bcc $\mathrm{Fe}(001)$. Therefore, at the zone center $\Gamma$ in the BZ, where $\left(k_{x}, k_{y}\right)=$ $(0,0)$, the bands $\Delta_{1}\left(s, p_{z}, d_{z^{2}}\right), \Delta_{2}\left(d_{x^{2}-y^{2}}\right), \Delta_{2}^{\prime}\left(d_{x y}\right)$, and $\Delta_{5}\left(p_{x}, p_{y}, d_{x z}, d_{y z}\right)$ defined by $\mathrm{Fe}(001)$ were transformed into the $\tilde{\Delta}_{1}\left(s, p_{z}, d_{z^{2}}, d_{x^{2}-y^{2}}\right), \quad \tilde{\Delta}_{2}\left(d_{x y}\right), \quad \tilde{\Delta}_{3}\left(p_{x}, d_{x z}\right)$, and $\tilde{\Delta}_{4}\left(p_{y}, d_{y z}\right)$ bands, respectively, in the spinel [42]. The calculated results in Fig. 8(c) show that at $\left(k_{x}, k_{y}\right)=(0,0)$, the real band structures of the conduction bottom and the valence top consist of the $\tilde{\Delta}_{1}$ states, where the former has $\mathrm{O}-s$ and $\mathrm{Ga}-s$ orbital weights and the latter has $\mathrm{O}-p_{z}$ and $\mathrm{Ga}-d_{z^{2}}$ orbital weights, with an energy band gap of $3.8 \mathrm{eV}$. In this gap, the real band of the valence top state is not connected to the conduction bottom state directly by the imaginary band, unlike the MAO-MTJ system, where the single imaginary band structure is connected directly to the real band structures of the valence top and the conduction bottom at $\Gamma$. [17,42] In contrast, the MGO has an imaginary band that is connected to both the real valence and conduction bands at $\left(k_{x}, k_{y}\right)=$ $(0,0.1)$. This observed tendency indicates that the coherent tunneling at $\Gamma$ may not provide the largest contribution to the spin-dependent transport in the MGO-MTJ.
[1] F. Hellman, A. Hoffmann, Y. Tserkovnyak, G. S. D. Beach, E. E. Fullerton, C. Leighton, A. H. MacDonald, D. C. Ralph, D. A. Arena, H. A. Dürr, P. Fischer, J. Grollier, J. P. Heremans, T. Jungwirth, A. V. Kimel, B. Koopmans, I. N. Krivorotov, S. J. May, A. K. Petford-Long, J. M. Rondinelli, N. Samarth, I. K. Schuller, A. N. Slavin, M. D. Stiles, O. Tchernyshyov, A. Thiaville, and B. L. Zink, Rev. Mod. Phys. 89, 025006 (2017), and references therein.
[2] S. Bhatti, R. Sbiaa, A. Hirohata, H. Ohno, S. Fukami, and S. N. Piramanayagam, Mater. Today 20, 530 (2017).

[3] Y. Ando, M. Hayashi, S. Iura, K. Yaoita, C. C. Yu, H. Kubota, and T. Miyazaki, J. Phys. D 35, 2415 (2002).

[4] K. Sin, M. Mao, C. Chien, S. Funada, L. Miloslavsky, H.-C. Tong, and S. Gupta, IEEE Trans. Magn. 36, 2818 (2000). 
[5] S. Kasai, Y. K. Takahashi, P.-H. Cheng, Ikhtiar, T. Ohkubo, K. Kondou, Y. Otani, S. Mitani, and K. Hono, Appl. Phys. Lett. 109, 032409 (2016).

[6] K. Masuda and Y. Miura, Jpn. J. Appl. Phys. 56, 020306 (2017).

[7] K. Masuda and Y. Miura, J. Magn. Soc. Jpn. 42, 37 (2018).

[8] K. Mukaiyama, J. W. Jung, H. Sepehri-Amin, S. Kasai, T. Furubayashi, T. Ohkubo, and K. Hono, Appl. Phys. Lett. 114, 172402 (2019).

[9] X. Jiang, A. F. Panchula, and S. S. P. Parkin, Appl. Phys. Lett. 83, 5244 (2003).

[10] T. Iwase, Y. Sakuraba, S. Bosu, K. Saito, S. Mitani, and K. Takanashi, Appl. Phys. Express 2, 063003 (2009).

[11] Y. Sakuraba, K. Izumi, T. Iwase, S. Bosu, K. Saito, K. Takanashi, Y. Miura, K. Futatsukawa, K. Abe, and M. Shirai, Phys. Rev. B 82, 094444 (2010).

[12] T. M. Nakatani, T. Furubayashi, S. Kasai, H. Sukegawa, Y. K. Takahashi, S. Mitani, and K. Hono, Appl. Phys. Lett. 96, 212501 (2010).

[13] Y. K. Takahashi, A. Srinivasan, B. Varaprasad, A. Rajanikanth, N. Hase, T. M. Nakatani, S. Kasai, T. Furubayashi, and K. Hono, Appl. Phys. Lett. 98, 152501 (2011).

[14] J. Sato, M. Oogane, H. Naganuma, and Y. Ando, Appl. Phys. Express 4, 113005 (2011).

[15] Y. Sakuraba, M. Udeda, Y. Miura, K. Sato, S. Bosu, K. Saito, M. Shirai, T. J. Konno, and K. Takanashi, Appl. Phys. Lett. 101, 252408 (2012).

[16] Y. Sakuraba, M. Ueda, S. Bosu, K. Saito, and K. Takanashi, J. Magn. Soc. Jpn. 38, 45 (2014).

[17] Y. Miura, S. Muramoto, K. Abe, and M. Shirai, Phys. Rev. B 86, 024426 (2012).

[18] H. Sukegawa, Y. Miura, S. Muramoto, S. Mitani, T. Niizeki, T. Ohkubo, K. Abe, M. Shirai, K. Inomata, and K. Hono, Phys. Rev. B 86, 184401 (2012).

[19] H. Sukegawa, K. Inomata, and S. Mitani, Appl. Phys. Lett. 105, 092403 (2014).

[20] H. Sukegawa, H. Xiu, T. Ohkubo, T. Furuyabashi, T. Niizeki, W. Wang, S. Kasai, S. Mitani, K. Inomata, and K. Hono, Appl. Phys. Lett. 96, 212505 (2010).

[21] M. Belmoubarik, H. Sukegawa, T. Ohkubo, S. Mitani, and K. Hono, AIP Adv. 7, 055908 (2017).

[22] H. Sukegawa, S. Mitani, T. Ohkubo, K. Inomata, and K. Hono, Appl. Phys. Lett. 103, 142409 (2013).

[23] M. Belmoubarik, H. Sukegawa, T. Ohkubo, S. Mitani, and K. Hono, Appl. Phys. Lett. 108, 132404 (2016).

[24] S. Ju, Y. Miura, K. Yamamoto, K. Masuda, K.-i. Uchida, and J. Shiomi, Phys. Rev. Res. 2, 023187 (2020).

[25] Z. Galazka, D. Klimm, K. Irmscher, R. Uecker, M. Pietsch, R. Bertram, M. Naumann, M. Albrecht, A. Kwasniewski, R. Schewski, and M. Bickermann, Phys. Status Solidi A 212, 1455 (2015)

[26] M. L. Bortz, R. H. French, D. L. Jones, R. V. Kasowski, and F. S. Ohuchi, Phys. Scr. 41, 537 (1990).

[27] M. L. Bortz and R. H. French, Appl. Phys. Lett. 55, 1955 (1989).

[28] R. H. French, J. Am. Ceram. Soc. 73, 477 (1990).

[29] D. M. Roessler and W. C. Walker, Phys. Rev. 159, 733 (1967).

[30] H. Sukegawa, Y. Kato, M. Belmoubarik, P.-H. Cheng, T. Daibou, N. Shimomura, Y. Kamiguchi, J. Ito, H. Yoda, T. Ohkubo, S. Mitani, and K. Hono, Appl. Phys. Lett. 110, 122404 (2017).
[31] A. Smogunov, A. Dal Corso, and E. Tosatti, Phys. Rev. B 70 , 045417 (2004).

[32] P. Giannozzi, S. Baroni, N. Bonini, M. Calandra, R. Car, C. Cavazzoni, D. Ceresoli, G. L. Chiarotti, M. Cococcioni, I. Dabo et al., J. Phys.: Condens. Matter 21, 395502 (2009).

[33] P. Giannozzi, O. Andreussi, T. Brumme, O. Bunau, M. B. Nardelli, M. Calandra, R. Car, C. Cavazzoni, D. Ceresoli, M. Cococcioni et al., J. Phys.: Condens. Matter 29, 465901 (2017).

[34] J. P. Perdew, K. Burke, and M. Ernzerhof, Phys. Rev. Lett. 77, 3865 (1996).

[35] H. J. Choi and J. Ihm, Phys. Rev. B 59, 2267 (1999).

[36] The barrier thickness is defined as $t=\left(t_{\text {barrier }}+d_{\text {electrode }}\right) / 2$, where $t_{\text {barrier }}\left(d_{\text {electrode }}\right)$ is the maximum (minimum) distance between the $\mathrm{O}$ of the spinel MGO (between the $\mathrm{Fe}$ of the electrode) layers along the $z$ axis.

[37] C. Heiliger, P. Zahn, B. Y. Yavorsky, and I. Mertig, Phys. Rev. B 77, 224407 (2008)

[38] W. H. Butler, X.-G. Zhang, T. C. Schulthess, and J. M. MacLaren, Phys. Rev. B 63, 054416 (2001).

[39] J. Mathon and A. Umerski, Phys. Rev. B 63, 220403(R) (2001).

[40] S. S. P. Parkin, C. Kaiser, A. Panchula, P. M. Rice, B. Hughes, M. Samant, and S.-H. Yang, Nat. Mater. 3, 862 (2004).

[41] S. Yuasa, T. Nagahama, A. Fukushima, Y. Suzuki, and K. Ando, Nat. Mater. 3, 868 (2004).

[42] J. Zhang, X.-G. Zhang, and X. F. Han, Appl. Phys. Lett. 100, 222401 (2012).

[43] K. Masuda and Y. Miura, Phys. Rev. B 96, 054428 (2017).

[44] K. D. Belashchenko, J. Velev, and E. Y. Tsymbal, Phys. Rev. B 72, 140404(R) (2005).

[45] X.-G. Zhang, W. H. Butler, and A. Bandyopadhyay, Phys. Rev. B 68, 092402 (2003).

[46] D. Wortmann, G. Bihlmayer, and S. Blügel, J. Phys.: Condens. Matter 16, S5819 (2004).

[47] C. Tusche, H. L. Meyerheim, N. Jedrecy, G. Renaud, A. Ernst, J. Henk, P. Bruno, and J. Kirschner, Phys. Rev. Lett. 95, 176101 (2005).

[48] A. L. Allred, J. Inorg. Nucl. Chem. 17, 215 (1961).

[49] We find that the interfacial distances at the spinel/Fe interfaces are almost the same for the MTJs with the MGO and MAO barriers. The averaged distance of the MGO-MTJ is $1.951 \AA$, while that of the MAO-MTJ is $1.989 \AA$. Therefore, we speculate based on the discussion available in the main text that the difference in the $x y$-plane shift of the anions $(\mathrm{O})$ between the MGOand MAO-MTJs is more significant than the corresponding difference in the $z$-axis shift.

[50] X.-G. Zhang and W. H. Butler, Phys. Rev. B 70, 172407 (2004).

[51] D. Waldron, V. Timoshevskii, Y. Hu, K. Xia, and H. Guo, Phys. Rev. Lett. 97, 226802 (2006).

[52] I. Rungger, A. R. Rocha, O. Mryasov, O. Heinonen, and S Sanvito, J. Magn. Magn. Mater. 316, 481 (2007).

[53] J. P. Trinastic, Y. Wang, and H.-P. Cheng, Phys. Rev. B 88, 104408 (2013).

[54] K. Masuda, H. Itoh, and Y. Miura, Phys. Rev. B 101, 144404 (2020).

[55] K. Masuda, H. Itoh, Y. Sonobe, H. Sukegawa, S. Mitani, and Y. Miura, arXiv:2007.01068.

[56] Y. Wang, X. F. Han, and X.-G. Zhang, Appl. Phys. Lett. 93, 172501 (2008). 
[57] K. Yamamoto, K. Masuda, K.-i. Uchida, and Y. Miura, Phys. Rev. B 101, 094430 (2020).

[58] The effect of strain on the Seebeck effect in the $\mathrm{Fe} / \mathrm{MgO} / \mathrm{Fe}$ MTJ was investigated but the TMR was not studied in Ref. [57]. However, it is visible from Fig. 4 of Ref. [57] that the resonance behavior of the minority spin exists in the transmittance around the Fermi energy when the in-plane lattice is compressively distorted, whereas there is an absence of these resonance states when the lattice is distorted extensively.

[59] C. Tiusan, J. Faure-Vincent, C. Bellouard, M. Hehn, E. Jouguelet, and A. Schuhl, Phys. Rev. Lett. 93, 106602 (2004).
[60] I. Rungger, O. Mryasov, and S. Sanvito, Phys. Rev. B 79, 094414 (2009).

[61] T. F. W. Barth and E. Posnjak, Z. Kristallogr. 82, 325 (1932).

[62] M. Huber, C. R. Acad. Sci., Paris 244, 2524 (1957).

[63] F. Machatschki, Z. Kristallogr. 82, 348 (1932).

[64] J. W. Weidenborner, N. R. Stemple, and Y. Okaya, Acta Crystallogr. 20, 761 (1966).

[65] H. Schmalzried, Z. Phys. Chem. 28, 203 (1961).

[66] A. Seko, K. Yuge, F. Oba, A. Kuwabara, and I. Tanaka, Phys. Rev. B 73, 184117 (2006).

[67] S.-H. Wei and S. B. Zhang, Phys. Rev. B 63, 045112 (2001).

[68] K. Masuda and Y. Miura, Phys. Rev. B 98, 224421 (2018). 